\title{
Bickerstaff encephalitis after COVID-19
}

\author{
Lucía Llorente Ayuso ${ }^{1}$ Pedro Torres Rubio ${ }^{2}$ - Rafael Fernando Beijinho do Rosário ${ }^{3}$. \\ María Luisa Giganto Arroyo ${ }^{4} \cdot$ Fernando Sierra-Hidalgo $^{1}$ (i)
}

Received: 12 July 2020 / Revised: 27 August 2020 / Accepted: 29 August 2020 / Published online: 3 September 2020

(c) Springer-Verlag GmbH Germany, part of Springer Nature 2020

\section{Dear Sirs,}

A 72-year-old woman with a history of hypertension, hyperlipidemia, smoking, and depression presented on early April 2020 with delirium and fever. A cranial CT scan was normal. A chest X-ray showed bilateral interstitial pneumonia, and nasopharyngeal exudate polymerase chain reaction (PCR) testing was positive to SARS-CoV-2. Cerebrospinal fluid (CSF) was normal. She was admitted and started on hydroxychloroquine, azithromycin, ceftriaxone, and IV methylprednisolone. A few days later she was transferred to the intensive care unit due to a cardiogenic shock caused by a myocardial infarction. Further hemodynamic and respiratory evolution was good, and she was discharged without delirium or cognitive impairment on day 22 after admission.

She was readmitted eight days later due to a 48-h history of dizziness, oscillopsia, and unsteadiness. Her vital signs were normal, and she was afebrile. Systemic examination was unremarkable. She was conscious, and her language and speech were normal, but slight inattention and disorientation were present. A downbeat nystagmus in all gaze positions and impairment of smooth pursuit eye movements were present. Horizontal and vertical eye movements showed no limitation. Motor and sensory examinations were normal, and deep tendon reflexes were all present and symmetrical. The left plantar response was extensor. There was no limb dysmetria, but severe truncal ataxia was present. Reflex

Fernando Sierra-Hidalgo

fernando.sierragh@salud.madrid.org

1 Department of Neurology, Hospital Universitario Infanta Leonor, Avenida Gran Vía del Este 80, 28031 Madrid, Spain

2 Department of Radiology, Unidad Central de Radiodiagnóstico, Hospital Universitario Infanta Leonor, Madrid, Spain

3 Emergency Department, Hospital Universitario Infanta Leonor, Madrid, Spain

4 Department of Cardiology, Hospital Universitario Infanta Leonor, Madrid, Spain myoclonus in the face and both arms could be induced by sound and tactile stimuli.

Upon admission, PCR to SARS-CoV-2 was negative. Brain magnetic resonance imaging (MRI) showed hyperintense lesions in the caudal vermis and right flocculus, and contrast enhancement was observed in the floor of the fourth ventricle (Fig. 1). An electroencephalogram was normal. The CSF examination then showed a leukocyte count of $0 / \mathrm{mm}^{3}$, glucose level of $70 \mathrm{mg} / \mathrm{dl}$, protein level of $41 \mathrm{mg} / \mathrm{dl}$, sterile cultures, IgG index of 0.5 , and the absence of oligoclonal bands. Blood serological tests for HIV and Treponema pallidum were negative, as well as IgM for Varicella-zoster virus, Epstein Barr virus, Cytomegalovirus, Mycoplasma pneumoniae, and Borrelia burgdorferi. Anti-glutamic acid decarboxylase, antithyroid, anti-transglutaminase, and antinuclear antibodies all tested negative. Electromyography was not performed. Anti-Hu, anti-Yo, anti-Ri, anti-CV2, anti-Ma2, and anti-amphiphysin antibodies were absent in serum and CSF.

Once other causes were excluded, a post-infectious, immune-mediated rhomboencephalitis was suspected, and the patient was treated with IV methylprednisolone $1,000 \mathrm{mg}$ daily for five days, followed by $1 \mathrm{mg} / \mathrm{kg} /$ day of PO prednisone. A significant improvement existed within days, with resolution of bradypsychia, nystagmus, Babinski sign, and myoclonus, and improvement of ataxia.

Anti-GD1a IgG antibodies (immunoblot analysis) tested positive in serum. A follow-up MRI performed on day 24 after admission revealed a significant decrease of the vestibulocerebellar hyperintensities, and the patient was discharged. Only a very mild unsteadiness persisted in a followup visit two months later.

This patient had an acute brainstem and cerebellar dysfunction that appeared within several days after getting over COVID-19. The presence of a previous viral disease, pyramidal tract involvement, brainstem enhancement on MRI, and positive testing for anti-GD1a antibodies led to a diagnosis of Bickerstaff brainstem encephalitis (BBE) despite the absence of ophthalmoplegia $[1,2]$. 

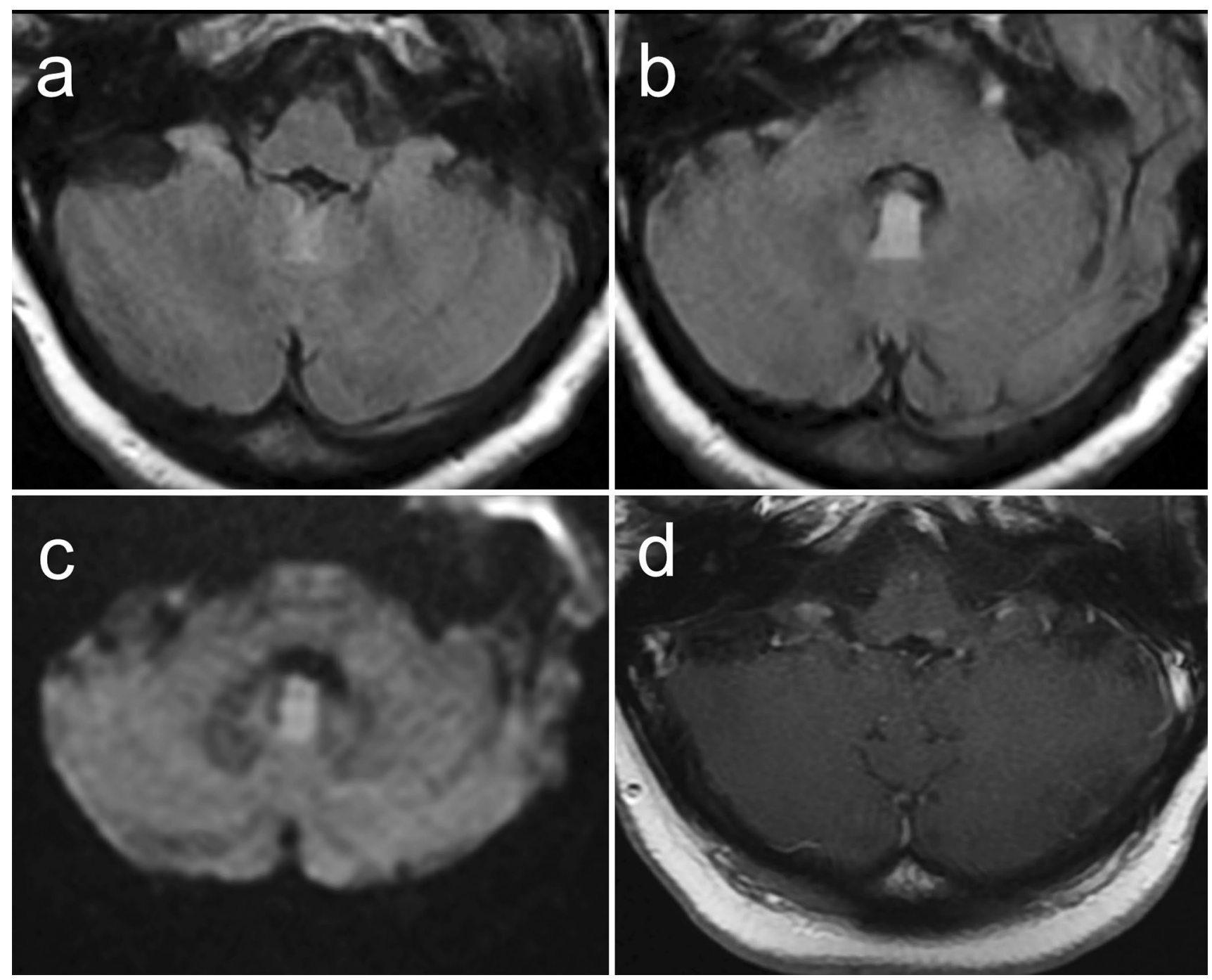

Fig. 1 Brain MRI findings. a and $\mathbf{b}$, high signal intensity of the right flocculus and the nodulus on FLAIR images. $\mathbf{c}$ weak restriction of the nodulus on diffusion-weighted images. d lineal gadolinium enhancement of dorsal medulla on T1-weighted images

Anti-GD1a antibodies are well recognized in BBE, and cerebellum may be also involved in antiganglioside antibodies syndromes [3, 4]. Increasing evidence suggests that an enhanced immune response is involved in the physiopathology of a wide range of complications of SARSCoV-2 infection, including neurological disorders [5]. Guillain-Barré syndrome, Miller Fisher syndrome, acute necrotizing encephalitis, myelitis, acute disseminated encephalomyelitis (ADEM), and myasthenia gravis have been reported after COVID-19 [6-12]. As in the described patient, immunotherapy seemed to have a beneficial effect in several of these cases. BBE widens the spectrum of para- or post-infectious neurological disorders related to COVID-19.

Funding None.

\section{Compliance with ethical standards}

Conflicts of interest On behalf of all authors, the corresponding author states that there is no conflict of interest.

Ethical standards This report belongs to the COVID-19@ Vallecas cohort. The study was approved by the Hospital Universitario Infanta Leonor ethics committee and has therefore been performed in accordance with the ethical standards laid down in the 1964 Declaration of Helsinki and its later amendments.

Consent to participate Written informed consent was obtained.

Availability of data and material Data of the reported case is presented within the manuscript. 


\section{References}

1. Wakerley BR, Soon D, Chan YC, Yuki N (2013) Atypical Bickerstaff brainstem encephalitis: ataxic hypersomnolence without ophthalmoplegia. J Neurol Neurosurg Psychiatry 84:1206-1207. https://doi.org/10.1136/jnnp-2013-304993

2. Kuwabara S, Misawa S, Mori M (2013) Bickerstaff brainstem encephalitis: more common than we think? J Neurol Neurosurg Psychiatry 84:1184. https://doi.org/10.1136/jnnp-2013-305239

3. Odaka M, Yuki N, Yamada M et al (2003) Bickerstaff's brainstem encephalitis: clinical features of 62 cases and a subgroup associated with Guillain-Barré syndrome. Brain 126:2279-2290. https ://doi.org/10.1093/brain/awg233

4. Lee SU, Kim HJ, Choi JY, Kim JK, Kim JS (2019) Acute vestibular syndrome associated with anti-GQ1b antibody. Neurology 93:e1085-e1092. https://doi.org/10.1212/WNL.0000000000 008107

5. Reichard RR, Kashani KB, Boire NA, Constantopoulos E, Guo Y, Lucchinetti CF (2020) Neuropathology of COVID-19: a spectrum of vascular and acute disseminated encephalomyelitis (ADEM)-like pathology. Acta Neuropathol 140:1-6. https://doi. org/10.1007/s00401-020-02166-2

6. Alberti P, Beretta S, Piatti M et al (2020) Guillain-Barré syndrome related to COVID-19 infection. Neurol Neuroimmunol
Neuroinflamm 7:e741. https://doi.org/10.1212/NXI.0000000000 000741

7. Gutiérrez-Ortiz C, Méndez A, Rodrigo-Rey S et al (2020) Miller Fisher Syndrome and polyneuritis cranialis in COVID-19. Neurology. https://doi.org/10.1212/WNL.0000000000009619

8. Poyiadji N, Shahin G, Noujaim D, Stone M, Patel S, Griffith B (2020) COVID-19-associated acute hemorrhagic necrotizing encephalopathy: CT and MRI features. Radiology. https://doi. org/10.1148/radiol.2020201187

9. Parsons T, Banks S, Bae C, Gelber J, Alahmadi H, Tichauer M (2020) COVID-19-associated acute disseminated encephalomyelitis (ADEM). J Neurol. https://doi.org/10.1007/s00415-020-09951 $-9$

10. Romero-Sánchez CM, Díaz-Maroto I, Fernández-Díaz E et al (2020) Neurologic manifestations in hospitalized patients with COVID-19: the ALBACOVID registry. Neurology. https://doi. org/10.1212/WNL.0000000000009937

11. Paterson RW, Brown RL, Benjamin L et al (2020) The emerging spectrum of COVID-19 neurology: clinical radiological and laboratory findings. Brain. https://doi.org/10.1093/brain/awaa240

12. Restivo DA, Centonze D, Alesina A, Marchese-Ragona R (2020) Myasthenia gravis associated with SARS-CoV-2 infection. Ann Intern Med. https://doi.org/10.7326/L20-0845 